\title{
Type of Milk Feeding and Introduction to Complementary Foods in Relation to Infant Sleep: A Systematic Review
}

\author{
Xiaoxi Fu ${ }^{1} \mathbb{D}$, Amy L. Lovell ${ }^{1}\left(\mathbb{D}\right.$, Andrea J. Braakhuis ${ }^{1}\left(\mathbb{D}\right.$, Richard F. Mithen $^{2}$ and Clare R. Wall $^{1, *(D)}$ \\ 1 Department of Nutrition and Dietetics, Faculty of Medical and Health Sciences, University of Auckland, \\ Auckland 1142, New Zealand; xiaoxi.fu@auckland.ac.nz (X.F.); a.lovell@auckland.ac.nz (A.L.L.); \\ a.braakhuis@auckland.ac.nz (A.J.B.) \\ 2 The Liggins Institute, University of Auckland, Auckland 1142, New Zealand; r.mithen@auckland.ac.nz \\ * Correspondence: c.wall@auckland.ac.nz
}

check for

updates

Citation: Fu, X.; Lovell, A.L.; Braakhuis, A.J.; Mithen, R.F.; Wall, C.R. Type of Milk Feeding and Introduction to Complementary Foods in Relation to Infant Sleep: A Systematic Review. Nutrients 2021, 13, 4105. https://doi.org/10.3390/ nu13114105

Academic Editor: Raffaele Antonelli Incalzi

Received: 23 September 2021 Accepted: 13 November 2021 Published: 16 November 2021

Publisher's Note: MDPI stays neutral with regard to jurisdictional claims in published maps and institutional affiliations.

Copyright: (c) 2021 by the authors. Licensee MDPI, Basel, Switzerland. This article is an open access article distributed under the terms and conditions of the Creative Commons Attribution (CC BY) license (https:// creativecommons.org/licenses/by/ $4.0 /)$.

\begin{abstract}
Inconsistent conclusions from infant sleep and feeding studies may influence parents feeding-related decisions. This study aimed to systematically review the existing literature on infant sleep and its relation to the timing of introduction to complementary foods and type of milk feeding to better understand their role(s) in infant sleep. Cohort, longitudinal, cross-sectional studies, and controlled trials were identified using online searches of five databases up to April 2020. Twenty-one articles with a total of 6225 infants under 12 months-of-age were eligible. Exclusively breastfed infants ( $\leq 6$ months-of-age) had a greater number of night wakings, but most studies $(67 \%)$ reported no difference in night-time and $24 \mathrm{~h}$ sleep duration compared to formula-fed infants. However, after 6 months-of-age, most studies ( $>65 \%$ ) reported breastfed infants to sleep less in the night-time and over $24 \mathrm{~h}$ compared to formula-fed infants. Furthermore, studies reported no association between the timing of introduction to complementary foods and infant sleep duration ( $<12$ months-of-age). Future studies using standardized methodologies and definitions, transdisciplinary expertise, and longitudinal design are required to better understand the complex role of feeding on sleep.
\end{abstract}

Keywords: infant; 12 months and under; infant feeding mode; sleep; complementary feeding; night wakings; sleep duration; night-time sleep; systematic review

\section{Introduction}

Feeding type and sleep patterns are dynamic processes throughout the first year of life and have significant effects on health and development [1,2]. The World Health Organization (WHO) recommends exclusive breastfeeding for the first completed six months of life, with the introduction of complementary foods after six months-of-age [2]. Breastfeeding provides short- and long-term benefits to both infants and mothers, including protection against acute and chronic disorders among infants and as they grow older [3,4]. Sleep during the first year of life is especially important due to the rapid changes that occur in the consolidation of sleep/wake patterns [5-7]. The quantity and quality of an infant's sleep are associated with cognitive function such as the development of memory and language [8], and the ability to learn [9,10]. In addition, insufficient sleep and sleep problems have been associated with later obesity [11] and behavioral issues such as tantrums and other behavioral management problems [12]. Frequent and extended night wakings, one of the most common infant-sleep-related problems, has also been shown to affect infant health and development [13-15]. Therefore, sufficient sleep during infancy is a priority [8] and is often one of the main issues reported by new parents, with frequent parental night wakings shown to affect parent mood and function [13-16]. An estimated 20-30\% of children experience sleep problems during the first three years of life according to a cross-sectional study conducted in New Zealand and Australia, with one-third of parents reporting their infants as having a sleep problem [17]. 
A number of studies have examined the relationship between sleep and feeding among infants. The timing of introduction to complementary foods has been associated with infant sleep patterns, with breastfeeding reportedly playing a role in increasing sleep disturbances [17-21], while other studies have not found such significance [22,23]. The potential association between type of milk feeding or the timing of introduction to complementary foods and sleep may drive parental beliefs that early introduction to complementary foods or changes to the type of milk feeding, contrary to current recommendations [2], may improve their infants' sleep patterns [19,24]. The lack of consistency of the available evidence could be a source of confusion for parents, thereby affecting feeding-related decisions during the first year of life.

No systematic review has been completed to determine any associations between the timing of introduction to complementary foods and type of milk feeding on sleep in infants 12 months-of-age and younger. Therefore, this review aimed to systematically evaluate the existing literature to increase our understanding of this topic.

\section{Materials and Methods}

The review was registered with PROSPERO, the International Prospective Register of Systematic Reviews (Ref: CRD42020172830), which documents the inclusion and exclusion criteria for the review. Study selection and data were collected according to the 2009 Preferred Reporting Items for Systematic Reviews and Meta-Analyses (PRISMA) guidelines [25] and are displayed in Figure 1.

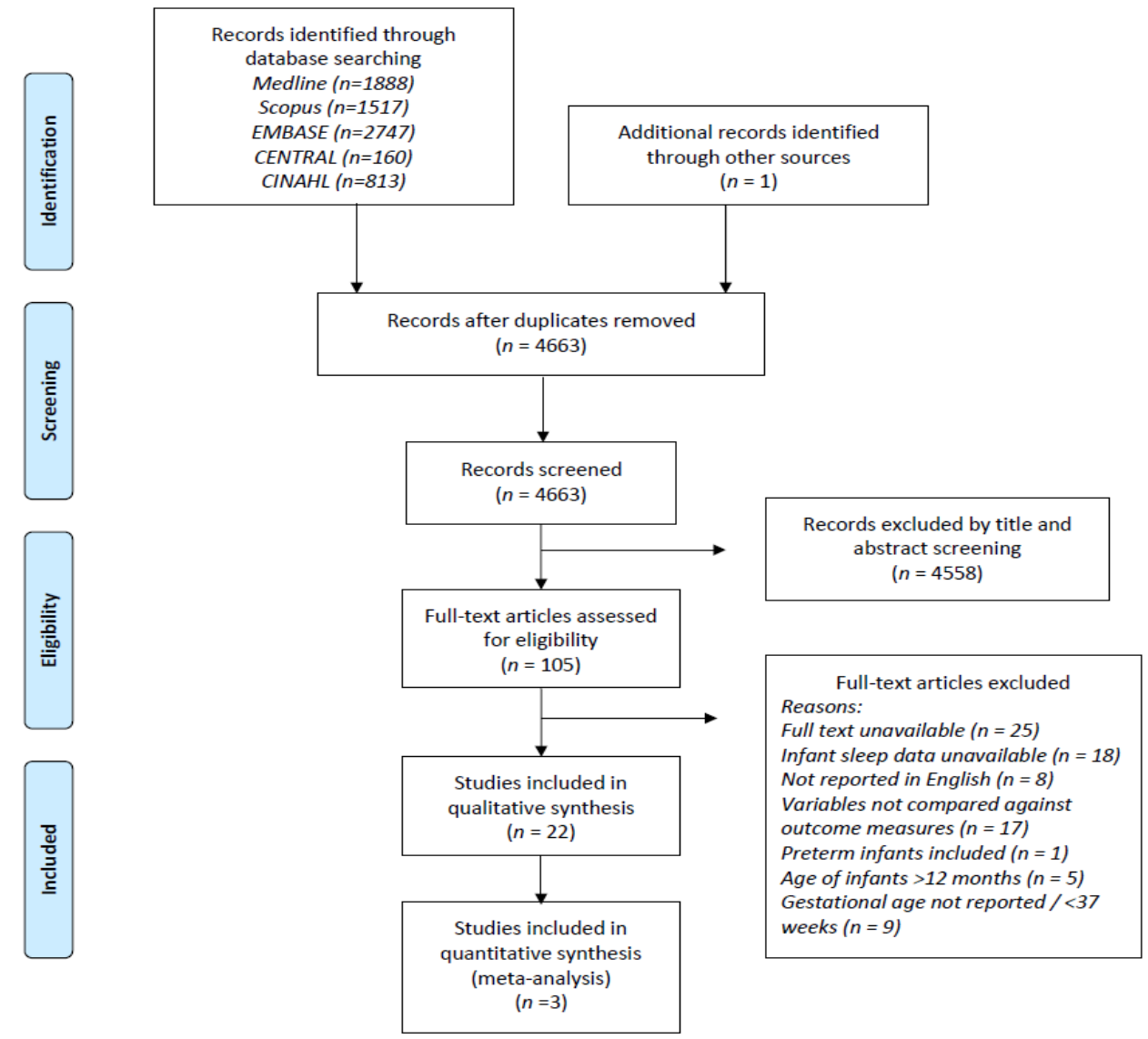

Figure 1. Preferred Reporting Items for Systematic Reviews and Meta-Analyses (PRIMSA) flow chart. The PRISMA flow chart is used for identification and selection of articles to be included for systematic review. A total of 7125 abstracts were retrieved using the search strategy. After removal of duplications, 4663 were screened by title and abstract, leaving 105 studies eligible for full-text review. Among the eligible studies, 84 articles were excluded due to the reasons listed. The remaining 21 studies were included for qualitative synthesis. 


\subsection{Search Strategy}

A search strategy (Supplementary Material, Table S1) was developed to identify studies that measured sleep, with the timing of introduction to complementary foods or type of milk feeding in infants 12 months-of-age and under. A preliminary search was conducted in March 2020, with the final search completed in five electronic databases (Ovid Medline, Ovid Embase, Scopus, Cochrane library (CENTRAL), and CINAHL) in April 2020. Medical Subject Heading (MeSH) terms and free terms were used for all databases, and a combination of subject headings and keywords were used for Ovid Medline and Embase. Only studies in English were included, and publication date restriction was not applied. Hand searches and independent reviews of reference lists were carried out to explore potentially eligible studies.

Studies included infants aged 12 months and younger, healthy, born at term $(\geq 37$ weeks gestation), measured infant sleep, and analyzed infant sleep data in relation to breastfeeding (and/or infant formula) or introduction to complementary foods, or both. Studies were excluded if infants were preterm ( $<37$ weeks), did not include any breastfeeding infants, investigated sleep by disease state, or if effects of a particular product or products were tested against infant sleep. Reviews (systematic, meta-analyses, and editorials without original data), qualitative studies, case studies, and animal studies were excluded.

\subsection{Selection of Studies}

Citations of the searched results from all five databases were downloaded into Endnote X9 citation management software (Clarivate Analytics, Philadelphia, USA) [26] and duplicates removed. One reviewer (X.F.) screened the study titles and abstracts using Endnote. Only studies that clearly did not meet the inclusion criteria were excluded during this stage, and all potentially relevant studies were retrieved for full-text review, which was carried out independently by X.F. and A.L.L. Screenings were based on predefined inclusion and exclusion criteria.

\subsection{Data Extraction and Quality Assessment}

The extraction form was developed by X.F. using examples in the relevant literature. The form was independently piloted by two reviewers (X.F. and A.L.L.) using the first five included studies. Adjustments were made, and the finalized form was used by X.F. and A.L.L. for independent data extraction on all included studies $(n=21)$. In the case of unresolved discrepancies, third (A.J.B.) and fourth (C.R.W.) reviewers were involved as the final decision makers. Relevant outcome data extracted included the mean, standard deviation or other variance data, and participant numbers in both control and intervention groups. Missing data were requested from corresponding authors via email. If no response was obtained from the authors, studies were excluded from the qualitative synthesis. Sleep data extracted included $24 \mathrm{~h}$ sleep duration, total night-time sleep, night-waking frequency, duration of night wakings, longest sleep period, and sleep onset latency. Qualitative synthesis was carried out with the assistance of tabulation of the results by grouping extracted data with similar outcomes within each age group ( $\leq 6$ months, $>6$ months, $0-12$ months). Criteria for meta-analysis were sleep variables with at least three independent studies that used a validated sleep assessment tool with available average and variance data deemed appropriate to pool. However, due to the high heterogeneity among the included studies, pooling the results was not possible, therefore meta-analysis was not deemed appropriate. Details on the heterogeneity of the included studies and inability to conduct a meta-analysis will be discussed in the limitations.

For studies evaluating the association between infant sleep and the type of milk feeding, standardized definitions for type of feeding were developed post hoc after study selection, deviating from the original PROSPERO protocol. This decision was made by the authors due to wide variation in the methodologies employed to categorize infants based on the type of milk feeding and timing of introduction to solids, as most studies were observational in design, with a lack of a comparator or control group. The agreed post hoc 
feeding groups were exclusive breastfeeding, breastfeeding, and formula feeding. Infants 6 months and younger categorized into the exclusive breastfeeding group had breastmilk only, as per the WHO definition [27]. Infants categorized into the breastfeeding group were partially or predominantly fed with breastmilk, and, infants categorized into the formula feeding group were exclusively or predominantly fed with infant formula. In the studies of infants 6 months and older, the following definitions were used to indicate if the infants had been introduced to complementary foods or not. Infants in the breastfeeding group were indicated as breastfeeding $+/$ - food as they were partially or predominantly breastfed with or without complementary foods. Infants in the formula feeding group were indicated as formula feeding $+/$ - food, who were predominantly formula-fed with the addition of, or exclusively formula-fed without the addition of, complementary foods. For studies that examined the association between infant sleep and the timing of introduction to complementary foods, infant age was recorded as reported (i.e., $\leq 12$ weeks vs. $>12$ weeks), and the above type of feeding definitions did not apply.

A quality assessment tool adapted from the Mixed Methods Appraisal Tool (MMAT, v.2018) [28] was used by two reviewers (X.F. and A.L.L.) to independently assess the quality of the included studies. The MMAT provides a checklist for describing and critically appraising included studies, providing a score that considers intra- and inter- individual variation. The questions from the checklist examined the representativeness of participants, appropriate measurements, complete outcome data, accounted confounders, and intended intervention administration. The tool was modified through the addition of the question "is the tool for measuring sleep validated or an objective assessment?". This question assessed the quality of sleep data extracted from the included studies. Following modification, the tool was scored out of 6 points (Supplementary Table S2). In this review, a low-quality study was defined by a score of 3 points and lower. Sensitivity analysis was carried out on sleep variables by comparing results before and after removal of the low-quality studies ( $\leq 3$ points).

\section{Results}

A total of 4662 studies were identified using the search results from all databases and other sources and after removal of duplicates (Figure 1). Table 1 summarizes the characteristics of all 21 studies included in the systematic review. Among these, nine (43\%) were cross-sectional [19,29-36], six (29\%) were cohort studies [23,37-41], five $(24 \%)$ were longitudinal studies [17,42-45], and one study combined randomized control trials [46]. The studies were mainly conducted in the United Kingdom $(n=5)$, and the United States $(n=8)$, making up more than half of the included studies. The remainder were from Israel $(n=2)$, Canada $(n=1)$, China $(n=2)$, Japan $(n=1)$, Portugal $(n=1)$, and South Korea $(n=1)$. The sample size of the included studies ranged from 20 to 1676 participants, totaling 6225 infants under 12 months-of-age. Subjective methods including sleep dairies/timetables, Brief Infant Sleep Questionnaire (BISQ), and other sleep questionnaires were used in 15 studies $[17,19,30-39,41,42,46]$ to assess sleep. The remaining six studies used objective methods to measure sleep, such as an actigraph or electroencephalogram (EEG). Four of these studies used a combination of subjective and objective measures, e.g., sleep diaries in conjunction with the actigraph (actimetry sensor) [40,43-45], one used a one channel (EEG) [29] and sleep diary, and another used EEG with a questionnaire [23] to capture infants' sleep patterns. 


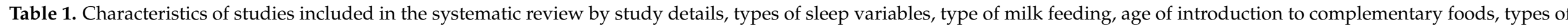
sleep assessment methods, and quality rating $(n=22)$.

\begin{tabular}{|c|c|c|c|c|c|c|c|}
\hline $\begin{array}{l}\text { Author, Year, } \\
\text { Country }\end{array}$ & Sample Size & Design & Sleep Variables & $\begin{array}{l}\text { Type of Milk } \\
\text { Feeding } 1\end{array}$ & $\begin{array}{l}\text { Age of Introduction to } \\
\text { Complementary Foods }{ }^{1}\end{array}$ & $\begin{array}{l}\text { Infant Sleep } \\
\text { Assessment }{ }^{1}\end{array}$ & Quality Rating $^{2}$ \\
\hline $\begin{array}{l}\text { Berger et al., 2017 } \\
\text { (United States) [37] }\end{array}$ & 57 & Prospective cohort & $\begin{array}{l}24 \text { h sleep duration } \\
\text { Night-time sleep } \\
\text { Night-waking frequency }\end{array}$ & $\begin{array}{l}\mathrm{BF} \\
\mathrm{FF}\end{array}$ & $x$ & BISQ & 6 \\
\hline $\begin{array}{l}\text { Brown et al., } 2015 \\
\text { (United } \\
\text { Kingdom) [19] }\end{array}$ & 756 & Cross-sectional & Night-waking frequency & $\begin{array}{l}\mathrm{BF}+/- \text { food } \\
\mathrm{FF}+/- \text { food }\end{array}$ & $\begin{array}{c}8 \text { to } 32 \text { weeks } \\
\text { (mean } 21.2 \text { weeks) }\end{array}$ & Questionnaire & 5 \\
\hline $\begin{array}{c}\text { Butte et al., } 1992 \\
\text { (United States) [29] }\end{array}$ & 20 & Cross-sectional & $\begin{array}{c}\text { Sleep diary: } \\
24 \text { h sleep duration } \\
\text { Night-time sleep } \\
\text { EEG: } \\
\text { Night-waking frequency } \\
\text { Sleep onset latency }\end{array}$ & $\begin{array}{l}\mathrm{BF} \\
\mathrm{FF}\end{array}$ & $x$ & $\begin{array}{l}5 \text { days sleep diary } \\
1 \text { night }^{3} \text { EEG }\end{array}$ & 5 \\
\hline $\begin{array}{l}\text { DeLeon et al., } 2007 \\
\text { (United States) [30] }\end{array}$ & 41 & Cross-sectional & $\begin{array}{c}\text { Duration of night wakings } \\
\text { Night-waking frequency } \\
\text { Night-time sleep }\end{array}$ & $\begin{array}{l}\mathrm{BF}+/- \text { food } \\
\mathrm{FF}+/- \text { food }\end{array}$ & $x$ & $\begin{array}{l}\text { Questionnaire and } \\
\text { sleep diary }\end{array}$ & 4 \\
\hline $\begin{array}{l}\text { Figueiredo et al., } 2017 \\
\text { (Portugal) [38] }\end{array}$ & $\begin{array}{c}148, \\
2 \text { weeks } \\
162 \\
3 \text { months } \\
123 \\
6 \text { months }\end{array}$ & Prospective cohort & $\begin{array}{l}\text { Night-time sleep } \\
\text { Night-waking frequency } \\
\text { Duration of night wakings } \\
\text { Sleep onset latency } \\
\text { Longest sleep period } \\
24 \text { h sleep duration }\end{array}$ & $\begin{array}{l}\text { EBF } \\
\text { BF } \\
\text { FF }\end{array}$ & $x$ & $\begin{array}{l}24 \mathrm{~h} \text { infant sleep } \\
\text { chronogram }\end{array}$ & 5 \\
\hline $\begin{array}{l}\text { Heinig et al., } 1993 \\
\text { (United States) [42] }\end{array}$ & 105 & Longitudinal study & $24 \mathrm{~h}$ sleep duration & $\begin{array}{l}\mathrm{BF}+/- \text { food } \\
\mathrm{FF}+/- \text { food }\end{array}$ & $\begin{array}{l}<26 \text { weeks } \\
\geq 26 \text { weeks }\end{array}$ & Sleep record & 4 \\
\hline $\begin{array}{l}\text { Huang et al., } 2016 \\
\text { (China) [39] }\end{array}$ & 524 & Prospective cohort & $\begin{array}{c}24 \mathrm{~h} \text { sleep } \% \\
\text { Night-waking frequency } \\
\text { night-time sleep \% } \\
\text { Longest sleep period }\end{array}$ & $\begin{array}{l}\mathrm{BF}+/- \text { food } \\
\mathrm{FF}+/- \text { food }\end{array}$ & $x$ & $\begin{array}{c}3 \text { and } 7 \text { days Sleep } \\
\text { diary } 4\end{array}$ & 4 \\
\hline $\begin{array}{l}\text { Kaley et al., } 2012 \\
\text { (United } \\
\text { Kingdom) [31] }\end{array}$ & 74 & Cross-sectional & $\begin{array}{l}\text { Night-waking frequency } \\
\text { Night-time sleep } \\
24 \text { h sleep duration }\end{array}$ & $\begin{array}{l}\mathrm{BF} \\
\mathrm{FF}\end{array}$ & $x$ & 3-7 days sleep diary & 4 \\
\hline
\end{tabular}


Table 1. Cont.

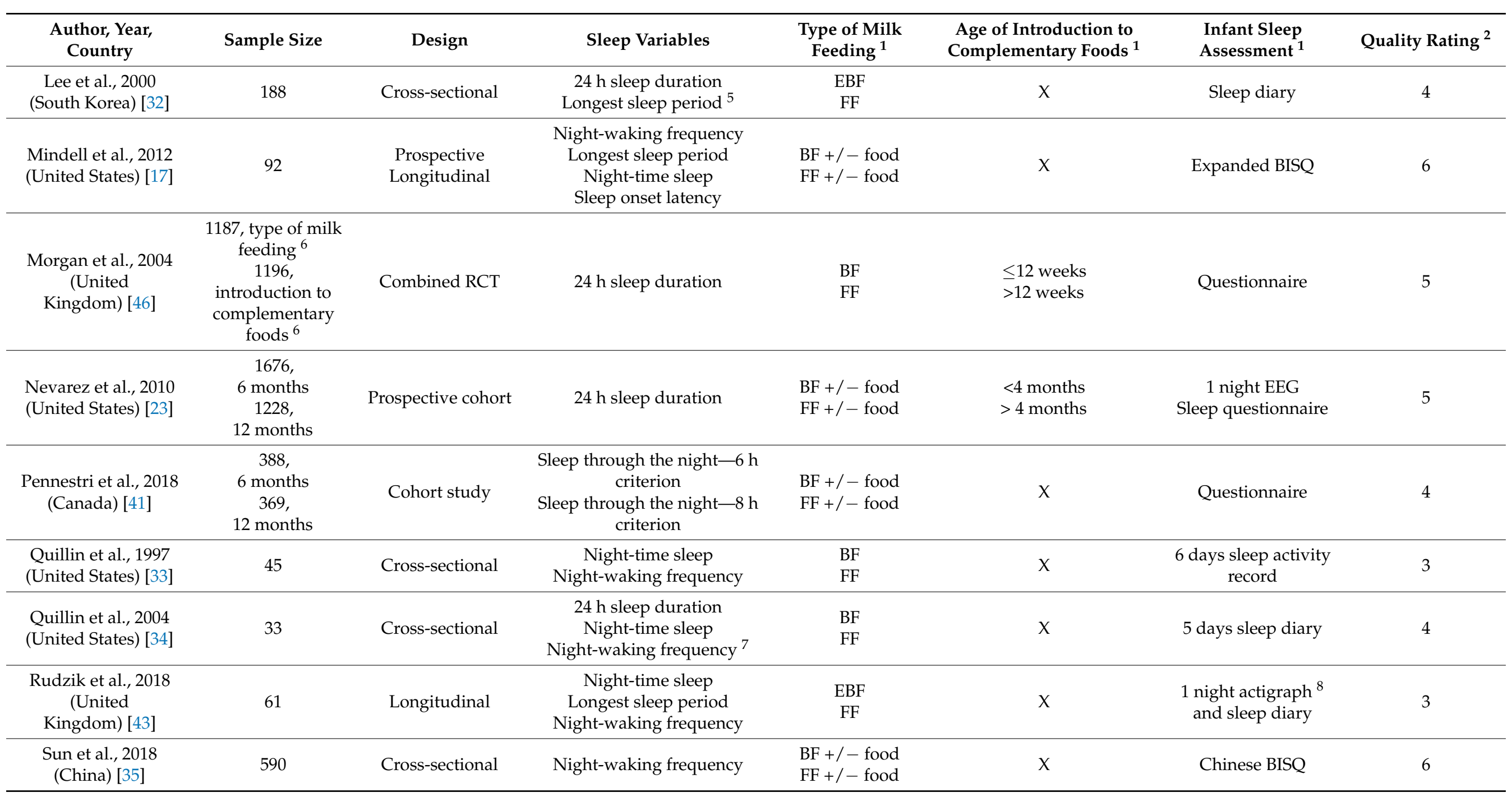


Table 1. Cont.

\begin{tabular}{|c|c|c|c|c|c|c|c|}
\hline $\begin{array}{l}\text { Author, Year, } \\
\text { Country }\end{array}$ & Sample Size & Design & Sleep Variables & $\begin{array}{l}\text { Type of Milk } \\
\text { Feeding }{ }^{1}\end{array}$ & $\begin{array}{l}\text { Age of Introduction to } \\
\text { Complementary Foods } 1\end{array}$ & $\begin{array}{l}\text { Infant Sleep } \\
\text { Assessment }^{1}\end{array}$ & Quality Rating ${ }^{2}$ \\
\hline $\begin{array}{l}\text { Tikotzky et al., } 2011 \\
\quad \text { (Israel) [44] }\end{array}$ & 56 & $\begin{array}{l}\text { Longitudinal } \\
\text { observational }\end{array}$ & $\begin{array}{l}\text { Night-waking frequency } \\
24 \text { h sleep duration }\end{array}$ & $\begin{array}{l}\text { EBF } \\
\text { BF } \\
\text { FF }\end{array}$ & $x$ & $\begin{array}{l}4 \text { days actigraph and } \\
\text { sleep diary }\end{array}$ & 6 \\
\hline $\begin{array}{l}\text { Tikotzky et al., } 2015 \\
\text { (Israel) [40] }\end{array}$ & 53 & Cohort & $\begin{array}{c}\text { Night-waking frequency }{ }^{9} \\
\text { Night-time sleep }{ }^{9}\end{array}$ & $\begin{array}{l}\text { EBF } \\
\text { BF } \\
\text { FF }\end{array}$ & $x$ & $\begin{array}{l}5 \text { days actigraph and } \\
\text { sleep diary, BISQ }\end{array}$ & 5 \\
\hline $\begin{array}{l}\text { Yoshida et al., } 2015 \\
\text { (Japan) [45] }\end{array}$ & 27 & Longitudinal & $\begin{array}{l}\text { Sleep through the night- } 6 \mathrm{~h} \\
\text { criterion } \\
\text { Duration of night wakings }\end{array}$ & $\begin{array}{l}\text { EBF } \\
\text { FF }\end{array}$ & $x$ & $\begin{array}{l}2 \text { days actigraph } \\
2 \text { days sleep } \\
\text { timetable }\end{array}$ & 5 \\
\hline
\end{tabular}

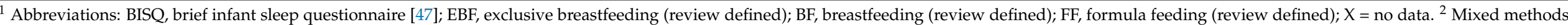

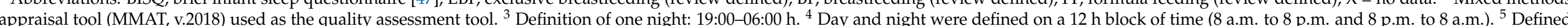

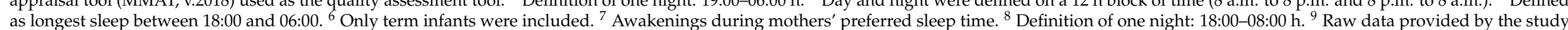
corresponding author, not found in the published manuscript 


\subsection{Type of Milk Feeding and Infant Sleep}

All twenty-one studies reported on type of milk feeding in relation to infant sleep patterns as shown in Table 2 . The type of milk feeding was reported prospectively by parents or caregivers through questionnaires $[17,19,23,30,31,35,38]$, interviews $[32,46]$, feeding logs [36,37,45], and by maternal self-report [33,34,39,40,43,44] except for one study [41], that assessed type of milk feeding retrospectively through a questionnaire. Two studies [29,42] did not specify their assessment methods.

\subsection{1. $24 \mathrm{~h}$ Sleep Duration}

Among infants aged 6 months and younger, exclusive breastfeeding was not associated with $24 \mathrm{~h}$ sleep duration in one study [44] and no difference in sleep duration compared to formula-feeding was reported in a cohort study [38]. However, one study reported significantly longer sleep duration in exclusively breastfed infants compared to formulafed infants [32]. There was no reported difference in sleep duration of breastfed infants compared to formula-fed infants in four studies $[23,29,31,38]$. In contrast, one study found that breastfed infants had significantly shorter sleep duration [34], while another study reported a significantly longer sleep duration compared to formula-fed infants [37].

Among infants older than 6 months, $24 \mathrm{~h}$ sleep duration did not differ between breastfed and formula-fed infants in one study [23], whilst two other studies reported significantly shorter sleep duration in breastfed infants compared to formula-fed infants [42,46].

Breastfeeding was associated with significantly shorter sleep duration compared to formula feeding in a study that examined infants 0 to 8 months-of-age [39].

\subsubsection{Total Night-Time Sleep}

Among infants aged 6 months and younger, no difference in total night-time sleep was reported between exclusively breastfed and formula-fed infants in one cohort and one longitudinal study, where sleep was measured at multiple time points [38,43]. However, one study reported exclusively breastfed infants at 3 and 4 months-of-age were less likely to sleep through the night compared to formula-fed infants [45]. There was no reported difference in total night-time sleep in breastfed infants compared to formula-fed infants in three studies [31,34,38]. However, three other studies reported a significantly shorter nighttime sleep duration experienced by breastfed infants [29,33,41], while one study reported breastfed infants to have a significantly longer night-time sleep duration compared to formula-fed infants [37].

Among infants older than 6 months, breastfeeding was inversely associated with night-time sleep duration in two studies [30,41].

No difference in night-time sleep duration was found in breastfed infants compared to formula-fed infants aged 3 to 12 months [17] and 0 to 8 months [39].

\subsubsection{Night-Waking Frequency}

In two studies, there was no reported differences in the frequency of night wakings in infants aged 6 months and younger who were exclusively breastfed or formula-fed [38,43]. However, one of these studies reported that infants that exclusively breastfed had significantly fewer night wakings at 16 weeks compared to formula-fed infants [43]. The majority of studies $(n=3)$ reported that exclusively breastfed infants had a significantly higher number of night-time wakings compared to formula-fed infants [38,40,44]. In contrast, the majority of the studies $(n=5)$ of breastfed infants and formula-fed infants reported no association $[29,34,36-38]$, with two studies reporting that breastfed infants had significantly more night-time wakings than formula-fed infants $[31,33]$. 


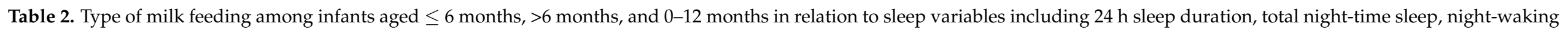
frequency, duration of night wakings, longest sleep period, and sleep onset latency.

\begin{tabular}{|c|c|c|c|c|c|c|c|}
\hline \multicolumn{8}{|c|}{24 h Sleep Duration } \\
\hline \multirow{2}{*}{ Author, Year } & \multirow{2}{*}{$\begin{array}{c}\text { Infant Age at } \\
\text { Assessment/Assessment } \\
\text { Frequency } \\
\end{array}$} & \multicolumn{2}{|c|}{$\leq 6$ Months } & \multirow{2}{*}{$\begin{array}{l}>6 \text { Months } \\
\text { BF vs. } \text { FF }^{1}\end{array}$} & \multirow{2}{*}{$\begin{array}{c}\text { 0-12 Months } \\
\text { BF vs. } \text { FF }^{1}\end{array}$} & \multirow{2}{*}{ Statistics ${ }^{1}$} & \multirow{2}{*}{ Quality Rating ${ }^{2}$} \\
\hline & & EBF vs FF ${ }^{1}$ & BF vs. FF ${ }^{1}$ & & & & \\
\hline Berger et al., 2017 [37] & 16 weeks & & $\begin{array}{c}\text { BF vs. FF } \\
\text { mean } \pm \text { SE }(\mathrm{h}) \\
12.95 \pm 0.51 \text { vs. } 11.43 \pm 0.53 \\
p=0.047\end{array}$ & & & $t$-test & 6 \\
\hline Butte et al., 1992 [29] & 17 weeks & & $\begin{array}{c}\text { BF vs. FF } \\
\text { mean } \pm \text { SD (h) } \\
13.2 \pm 2.3 \text { vs.13.3 } \pm 0.9, p>0.05\end{array}$ & & & $\begin{array}{c}t \text {-test } \\
\text { Regression }\end{array}$ & 5 \\
\hline Kaley et al., 2012 [31] & 4-10 weeks & & $\begin{array}{c}\text { BF vs. FF } \\
\text { Total sleep not assoc. with } \\
\text { feeding, } p>0.05\end{array}$ & & & $\begin{array}{l}\text { Correlation } \\
\text { ANOVA }\end{array}$ & 4 \\
\hline Lee et al., 2000 [32] & 2-17 weeks & $\begin{array}{c}\text { EBF vs. FF } \\
\text { mean } \pm \mathrm{SD}(\min ) \\
902.4 \pm 119.1 \text { vs. } 854.8 \pm \\
130.7, p<0.01\end{array}$ & & & & Unpaired $t$-test & 4 \\
\hline Quillin et al., 2004 [34] & 4 weeks & & $\begin{array}{c}B F \text { vs. } F F \\
\text { mean } \pm \mathrm{SD}(\mathrm{h}) \\
13.1 \pm 1.4 \text { vs. } 14.4 \pm 1.1 \\
p=0.006\end{array}$ & & & $t$-test & 4 \\
\hline Tikotzky et al., 2011 [44] & 26 weeks & $\begin{array}{c}\text { EBF vs. FF } \\
\text { No assoc. between total } \\
\text { sleep and EBF }(\mathrm{r}=0.15, \\
\quad p>0.05)\end{array}$ & & & & Spearman rho correlations & 6 \\
\hline Nevarez et al., 2010 [23] & 26,52 weeks ${ }^{4}$ & & $\begin{array}{c}\mathrm{BF}+/- \text { food vs. FF }+/- \text { food } \\
\text { Bivariate } \\
26 \text { weeks } \beta=0.05 \text { ( } 95 \% \mathrm{CI} \text { : } \\
-0.14 \text { to } 0.24), p>0.05 \\
\text { Multivariate } \\
26 \text { weeks } \beta=-0.15 \text { (95\%CI: } \\
-0.37 \text { to } 0.07), p>0.05\end{array}$ & $\begin{array}{c}\mathrm{BF}+/- \text { food vs. } \mathrm{FF}+/- \text { food } \\
\text { Bivariate } \\
52 \text { weeks } \beta=0.02(95 \% \mathrm{CI} \text { : } \\
-0.17 \text { to } 0.20), p>0.05 \\
\text { Multivariate } \\
52 \text { weeks is } \beta=-0.17 \text { ( } 95 \% \text { CI: } \\
-0.37 \text { to } 0.03), p>0.05\end{array}$ & & $\begin{array}{l}\text { Bivariate } \\
\text { Multivariate linear } \\
\text { regression }{ }^{5}\end{array}$ & 5 \\
\hline
\end{tabular}


Table 2. Cont.

\begin{tabular}{|c|c|c|c|c|c|c|c|}
\hline Heinig et al., 1993 [42] & 39 weeks & & & $\begin{array}{c}\mathrm{BF}+/- \text { food vs. FF }+/- \text { food } \\
24 \mathrm{~h} \text { sleep at } 39 \text { weeks greater in } \\
\text { FF compared to BF grps, } \\
p<0.05\end{array}$ & & $t$-test & 4 \\
\hline Morgan et al., 2004 [46] & 39 weeks ${ }^{4}$ & & & $\begin{array}{c}\mathrm{BF}+/- \text { food vs. } \mathrm{FF}+/- \text { food } \\
\text { mean } \pm \mathrm{SE}(\mathrm{h}) \\
11.2 \pm 0.1 \text { vs. }{ }_{6} .4 \pm 0.6, p=0.01\end{array}$ & & ANCOVA $^{7}$ & 5 \\
\hline Huang et al., 2016 [39] & $0-34$ weeks & & & . & $\begin{array}{c}\mathrm{BF}+/- \text { food vs. FF }+/- \\
\text { food } \\
\text { BF } 2.1 \% \text { lower (30 min less) } \\
24 \text { h sleep \% than FF, } \\
p=0.0009\end{array}$ & Multilevel mixed models & 4 \\
\hline \multicolumn{8}{|c|}{ Total Night-Time Sleep } \\
\hline \multirow{2}{*}{ Author, Year } & \multirow{2}{*}{$\begin{array}{c}\text { Infant Age at } \\
\text { Assessment/Assessment } \\
\text { Frequency } \\
\end{array}$} & \multicolumn{2}{|c|}{$\leq 6$ Months } & $>6$ Months & 0-12 Months & \multirow{2}{*}{ Statistics ${ }^{1}$} & \multirow{2}{*}{ Quality Rating } \\
\hline & & EBF vs. FF ${ }^{1}$ & BF vs. FF $^{1}$ & BF vs. FF $^{1}$ & BF vs. FF $^{1}$ & & \\
\hline Berger et al., 2017 [37] & 16 weeks & & $\begin{array}{c}\text { BF vs. FF } \\
\text { mean } \pm \text { SE }(h) \\
9.50 \pm 0.38 \text { vs. } 7.33 \pm 0.39 \\
p<0.0001\end{array}$ & & & $t$-test & 6 \\
\hline Butte et al., 1992 [29] & 17 weeks & & $\begin{array}{c}\text { BF vs. FF } \\
\text { mean } \pm \text { SD (h) } \\
8.2 \pm 1.6 \text { vs. } 9.9 \pm 1.4, p<0.04\end{array}$ & & & $\begin{array}{c}t \text {-test } \\
\text { Regression }\end{array}$ & 5 \\
\hline Figueiredo et al., 2017 [38] & $2,13,26$ weeks & $\begin{array}{c}\text { EBF vs. FF } \\
\text { mean } \pm \text { SD }(h) \\
2 \text { weeks } \\
7.08 \pm 1.33 \text { vs. } 6.34 \pm 1.21 \\
p>0.05 \\
13 \text { weeks } \\
8.06 \pm 1.30 \text { vs. } 8.27 \pm 1.35 \\
p>0.05 \\
26 \text { weeks } \\
8.29 \pm 1.36 \text { vs. } 8.29 \pm 1.07 \\
p>0.05\end{array}$ & $\begin{array}{c}\text { BF vs. FF } \\
\text { mean } \pm \text { SD }(h) \\
2 \text { weeks } \\
6.77 \pm 1.55 \text { vs. } 6.34 \pm 1.21 \\
p>0.05 \\
13 \text { weeks } \\
8.12 \pm 1.22 \text { vs. } 8.27 \pm 1.35 \\
p>0.05 \\
26 \text { weeks } \\
8.93 \pm 1.21 \text { vs. } 8.29 \pm 1.07 \\
p>0.05\end{array}$ & & & $\begin{array}{l}\text { Multivariate Analyses of } \\
\text { Chi MANCOVA }^{3}\end{array}$ & 5 \\
\hline Kaley et al., 2012 [31] & 4-10 weeks & & $\begin{array}{c}\text { BF vs. FF } \\
\text { NTS duration not assoc. with } \\
\text { feeding, } p>0.05\end{array}$ & & & $\begin{array}{l}\text { Correlation } \\
\text { ANOVA }\end{array}$ & 4 \\
\hline Quillin et al., 1997 [33] & 4 weeks & & $\begin{array}{c}\text { BF vs. FF } \\
\text { BF infants slept less at night } \\
\text { than FF infants. F }(1,39)=4.925, \\
p<0.05\end{array}$ & & & $\begin{array}{l}\text { ANOVA-two-way analysis } \\
\text { of variance }\end{array}$ & 3 \\
\hline Quillin et al., 2004 [34] & 4 weeks & & $\begin{array}{c}\text { BF vs. FF } \\
\text { mean } \pm \text { SD (h) } \\
6.4 \pm 1.0 \text { vs. } 6.4 \pm 0.8, p>0.05\end{array}$ & & & $t$-test & 4 \\
\hline
\end{tabular}


Table 2. Cont.

\begin{tabular}{|c|c|c|c|c|c|c|c|}
\hline Rudzik et al., 2018 [43] & $4,6,8,10,12,14,16,18$ weeks & $\begin{array}{c}\text { EBF vs. FF } \\
\text { Actigraph report } \\
\text { No difference between } \\
\text { grps for NTS at } 2,6,8,10 \text {, } \\
12,14,16,18 \text { weeks, } \\
p>0.05\end{array}$ & & & & $t$-test & 3 \\
\hline Yoshida et al., 2015 [45] & 13,17 weeks & $\begin{array}{c}\text { EBF vs. FF } \\
\text { STN (6 h criterion): } 33 \% \text { vs. } \\
67 \%\end{array}$ & & & & Multiple linear regression & 5 \\
\hline Pennestri et al., 2018 [41] & 26,52 weeks & & $\begin{array}{c}\mathrm{BF}+/- \text { food vs. FF }+/- \text { food } \\
\mathrm{BF} \text { infants less likely to STN at } \\
26 \text { weeks }\left(\chi^{2}=26.67, p<0.0001\right) \\
\text { using } 6 \mathrm{~h} \text { criterion } \\
\text { BF infants less likely to STN at } 6 \\
\text { months }\left(\mathrm{X}^{2}=31.19, p<0.0001\right) \\
\text { using } 8 \mathrm{~h} \text { criterion }\end{array}$ & $\begin{array}{c}\mathrm{BF}+/- \text { food vs. FF }+/- \text { food } \\
\mathrm{BF} \text { infants less likely to STN at } \\
52 \text { weeks }\left(\chi^{2}=34.96, p<0.0001\right) \\
\text { using } 6 \mathrm{~h} \text { criterion } \\
\text { BF infants less likely to STN at } \\
12 \text { months }\left(\chi^{2}=25.24\right. \\
p<0.0001) \text { using } 8 \mathrm{~h} \text { criterion } \\
\end{array}$ & & Chi-squared & 4 \\
\hline DeLeon et al., 2007 [30] & 39 weeks & & & $\begin{array}{c}\mathrm{BF}+/- \text { food vs. FF }+/- \text { food } \\
\text { BF }- \text { ve correlated with total } \\
\text { NTS }(\mathrm{r}=-0.42, p<0.01)\end{array}$ & & $\begin{array}{l}\text { Pearson's correlation } \\
\text { coefficient }\end{array}$ & 4 \\
\hline Huang et al., 2016 [39] & $0-34$ weeks & & & & $\begin{array}{c}\mathrm{BF}+/- \text { food vs. FF }+/- \\
\text { food } \\
\text { No assoc. between NTS \%, } \\
p>0.05\end{array}$ & Multilevel mixed models & 4 \\
\hline \multicolumn{8}{|c|}{ Night-Waking Frequency } \\
\hline \multirow{2}{*}{ Author, Year } & \multirow{2}{*}{$\begin{array}{c}\text { Infant Age at } \\
\text { Assessment/Assessment } \\
\text { Frequency }\end{array}$} & \multicolumn{2}{|c|}{$\leq 6$ Months } & $>6$ Months & 0-12 Months & \multirow{2}{*}{ Statistics ${ }^{1}$} & \multirow{2}{*}{ Quality Rating ${ }^{2}$} \\
\hline & & EBF vs. FF ${ }^{1}$ & BF vs. FF $^{1}$ & BF vs. FF $^{1}$ & BF vs. FF $^{1}$ & & \\
\hline Berger et al., 2017 [37] & 16 weeks & & $\begin{array}{c}\text { BF vs. FF } \\
\text { No difference in no. of NW, } \\
p>0.05\end{array}$ & & & $t$-test & 6 \\
\hline Butte et al., 1992 [29] & 17 weeks & & $\begin{array}{c}\text { BF vs. FF } \\
\text { mean } \pm \text { SD (no.) } \\
2.9 \pm 1.8 \text { vs. } 2.7 \pm 2.0, p>0.05\end{array}$ & & & $\begin{array}{c}t \text {-test } \\
\text { Regression }\end{array}$ & 5 \\
\hline
\end{tabular}


Table 2. Cont.

\begin{tabular}{|c|c|c|c|c|c|c|}
\hline Figueiredo et al., 2017 [38] & $2,13,26$ weeks & $\begin{array}{c}\text { EBF vs. FF } \\
\text { mean } \pm \text { SD (no.) } \\
2 \text { weeks } \\
3.02 \pm 0.83 \text { vs. } 2.96 \pm 0.88 \\
p>0.05 \\
13 \text { weeks } \\
2.19 \pm 1.07 \text { vs. } 1.65 \pm 1.17 \\
p>0.05 \\
26 \text { weeks } \\
2.22 \pm 1.01 \text { vs. } 1.53 \pm 0.90 \\
p<0.01\end{array}$ & $\begin{array}{c}\text { BF vs. FF } \\
\text { mean } \pm \text { SD (no.) } \\
2 \text { weeks } \\
2.63 \pm 0.67 \text { vs. } 2.96 \pm 0.88 \\
p>0.05 \\
13 \text { weeks } \\
2.18 \pm 1.36 \text { vs. } 1.65 \pm 1.17 \\
p>0.05 \\
26 \text { weeks } \\
1.73 \pm 0.94 \text { vs. } 1.53 \pm 0.90 \\
p>0.05\end{array}$ & & $\begin{array}{l}\text { Multivariate Analyses of } \\
\text { Chi MANCOVA }^{3}\end{array}$ & 5 \\
\hline Kaley et al., 2012 [31] & $4-10$ weeks & & $\begin{array}{c}\text { BF vs. FF } \\
\text { BF woke more freq. than FF, } \\
p<0.05\end{array}$ & & $\begin{array}{l}\text { Correlation } \\
\text { ANOVA }\end{array}$ & 4 \\
\hline Quillin et al., 1997 [33] & 4 weeks & & $\begin{array}{c}\text { BF vs. FF } \\
\text { BF infants had more } \\
\text { awakenings } \mathrm{F}(1,39)=12.231, \\
p<0.01\end{array}$ & & $\begin{array}{l}\text { ANOVA-two-way analysis } \\
\text { of variance }\end{array}$ & 3 \\
\hline Quillin et al., 2004 [34] & 4 weeks & & $\begin{array}{c}\text { BF vs. FF } \\
\text { Mean } \pm \text { SD (no.) }{ }^{8} \\
2.2 \pm 0.8 \text { vs. } 2.0 \pm 0.9, p>0.05\end{array}$ & & $t$-test & 4 \\
\hline Rudzik et al., 2018 [43] & $\begin{array}{c}4,6,8,10,12,14,16,18 \\
\text { weeks }\end{array}$ & $\begin{array}{c}\text { EBF vs. FF } \\
\text { Actigraphy report } \\
\text { EBF has 2.1 less NW at } 16 \\
\text { weeks, } p=0.05 \\
\text { No difference between } \\
\text { grps for number of NW at } \\
4,6,8,10,12,14,18 \text { weeks }\end{array}$ & & & $t$-test & 3 \\
\hline Tikotzky et al., 2011 [44] & 26 weeks & $\begin{array}{c}\text { EBF vs. FF } \\
\text { EBF assoc. with more NW } \\
\text { (Actigraph) }(\mathrm{r}=0.32 \\
p<0.05) \\
\end{array}$ & & & Spearman rho correlations & 6 \\
\hline Tikotzky et al., 2015 [40] & 26 weeks & $\begin{array}{c}\text { EBF vs. FF } \\
\text { Mean } \pm \text { SD (no.) } \\
2.53 \pm 1.08 \text { vs. } 1.48 \pm 0.96 \\
p<0.05\end{array}$ & & & Spearman CC & 5 \\
\hline Wailoo et al., 1990 [36] & 13-17 weeks & & $\begin{array}{c}\text { BF vs. FF } \\
\text { No difference in no. of NW, } \\
p>0.05\end{array}$ & & $t$-test & 2 \\
\hline Brown et al., 2015 [19] & $26-52$ weeks & & & $\begin{array}{c}\mathrm{BF}+/- \text { food vs. } \mathrm{FF}+/- \text { food } \\
\text { No difference in total NW } \\
\mathrm{F}(1711)=0.931, p=0.335\end{array}$ & MANOVA ${ }^{10}$ & 5 \\
\hline DeLeon et al., 2007 [30] & 39 weeks & & & $\begin{array}{l}\mathrm{BF}+/- \text { food vs. } \mathrm{FF}+/- \text { food } \\
\mathrm{BF}+\text { ve correlated with NW } \\
\text { frequency }(\mathrm{r}=0.48, p<0.01)\end{array}$ & $\begin{array}{l}\text { Pearson's correlation } \\
\text { coefficient }\end{array}$ & 4 \\
\hline
\end{tabular}


Table 2. Cont.

\begin{tabular}{|c|c|c|c|c|c|c|c|}
\hline Huang et al., 2016 [39] & 0-34 weeks & & & & $\begin{array}{l}\mathrm{BF}+/- \text { food vs. FF }+/- \\
\text { food } \\
\text { BF no diff as compared to } \\
\text { FF for NW, } p=0.0700\end{array}$ & Multilevel mixed models & 4 \\
\hline Mindell et al., 2012 [17] & $13-52$ weeks $^{4}$ & & & & $\begin{array}{c}\mathrm{BF}+/- \text { food vs. FF }+/- \\
\text { food } \\
\text { mean } \pm \mathrm{SD} \text { (no.) } \\
1.63 \pm 1.24 \text { vs. } 0.94 \pm 0.87 \\
p=0.003\end{array}$ & MANCOVA & 6 \\
\hline Sun et al., 2018 [35] & $8-52$ weeks & & & & $\begin{array}{l}\mathrm{BF}+/- \text { food vs. } \mathrm{FF}+/- \\
\text { food } \\
\text { Freq. NW assoc. with BF (v } \\
=0.18, p=0.002)\end{array}$ & $\begin{array}{c}\text { Chi-squared } \\
t \text {-test }\end{array}$ & \\
\hline \multicolumn{8}{|c|}{ Duration of Night Wakings } \\
\hline \multirow{2}{*}{ Author, Year } & \multirow{2}{*}{$\begin{array}{l}\text { Infant Age at } \\
\text { Assessment/Assessment } \\
\text { Frequency }\end{array}$} & \multicolumn{2}{|c|}{$\leq 6$ Months } & $>6$ Months & 0-12 Months & \multirow{2}{*}{ Statistics ${ }^{1}$} & \multirow{2}{*}{ Quality Rating ${ }^{2}$} \\
\hline & & EBF vs. FF $^{1}$ & BF vs. FF $^{1}$ & BF vs. FF $^{1}$ & BF vs. FF $^{1}$ & & \\
\hline Figueiredo et al., 2017 [38] & $2,13,26$ weeks & $\begin{array}{c}\text { EBF vs. FF } \\
\text { mean } \pm \text { SD }(h) \\
2 \text { weeks } \\
3.87 \pm 1.13 \text { vs. } 4.38 \pm 1.18 \\
p>0.05 \\
13 \text { weeks } \\
3.03 \pm 1.16 \text { vs. } 3.05 \pm 1.20 \\
p>0.05 \\
26 \text { weeks } \\
2.86 \pm 1.01 \text { vs. } 2.87 \pm 1.12 \\
p>0.05 \\
\end{array}$ & $\begin{array}{c}\text { BF vs. FF } \\
\text { mean } \pm \text { SD }(h) \\
2 \text { weeks } \\
4.00 \pm 1.11 \text { vs. } 4.38 \pm 1.18 \\
p>0.05 \\
13 \text { weeks } \\
3.00 \pm 1.16 \text { vs. } 3.05 \pm 1.20 \\
p>0.05 \\
26 \text { weeks } \\
2.14 \pm 0.90 \text { vs. } 2.87 \pm 1.12 \\
p>0.05\end{array}$ & & & $\begin{array}{l}\text { Multivariate analyses of } \\
\text { Chi MANCOVA }^{3}\end{array}$ & 5 \\
\hline Yoshida et al., 2015 [45] & 13,17 weeks & $\begin{array}{c}\text { EBF vs. FF } \\
\text { EBF +ve correlated with } \\
\text { wake time at night, } p<0.01\end{array}$ & & & & Multiple linear regression & 5 \\
\hline DeLeon et al., 2007 [30] & 39 weeks & & & $\begin{array}{l}\mathrm{BF}+/- \text { food vs. FF }+/- \text { food } \\
\mathrm{BF}+\mathrm{ve} \text { correlated with duration } \\
\text { of NW }(\mathrm{r}=0.33, p<0.05)\end{array}$ & & $\begin{array}{l}\text { Pearson's correlation } \\
\text { coefficient }\end{array}$ & 4 \\
\hline
\end{tabular}


Table 2. Cont.

\begin{tabular}{|c|c|c|c|c|c|c|c|}
\hline \multicolumn{8}{|c|}{ Longest Sleep Period } \\
\hline \multirow{2}{*}{ Author, Year } & \multirow{2}{*}{$\begin{array}{c}\text { Infant Age at } \\
\text { Assessment/Assessment } \\
\text { Frequency }\end{array}$} & \multicolumn{2}{|c|}{$\leq 6$ Months } & \multirow{2}{*}{$\begin{array}{l}>6 \text { Months } \\
\text { BF vs. } \text { FF }^{1}\end{array}$} & \multirow{2}{*}{$\begin{array}{c}\text { 0-12 Months } \\
\text { BF vs. } \text { FF }^{1}\end{array}$} & \multirow[b]{2}{*}{ Statistics ${ }^{1}$} & \multirow{2}{*}{ Quality Rating ${ }^{2}$} \\
\hline & & EBF vs. FF $^{1}$ & BF vs. FF $^{1}$ & & & & \\
\hline Figueiredo et al., 2017 [38] & $2,13,26$ weeks & $\begin{array}{c}\text { EBF vs. FF } \\
\text { mean } \pm \text { SD (h) } \\
2 \text { weeks } \\
3.04 \pm 1.00 \text { vs. } 2.82 \pm 0.90 \\
p>0.05 \\
13 \text { weeks } \\
5.26 \pm 2.15 \text { vs. } 6.50 \pm 2.44 \\
p<0.05 \\
26 \text { weeks } \\
5.38 \pm 2.45 \text { vs. } 6.76 \pm 1.96, \\
p<0.05\end{array}$ & $\begin{array}{c}\text { BF vs. FF } \\
\text { mean } \pm \text { SD }(h) \\
2 \text { weeks } \\
3.38 \pm 1.12 \text { vs. } 2.82 \pm 0.90 \\
p>0.05 \\
13 \text { weeks } \\
5.74 \pm 2.31 \text { vs. } 6.50 \pm 2.44 \\
p>0.05 \\
26 \text { weeks } \\
6.98 \pm 2.58 \text { vs. } 6.76 \pm 1.96 \\
p>0.05\end{array}$ & & & $\begin{array}{l}\text { Multivariate analyses of } \\
\text { Chi MANCOVA }^{3}\end{array}$ & 5 \\
\hline Lee et al., 2000 [32] & 2-17 weeks & $\begin{array}{c}\text { EBF vs. FF } \\
\text { mean } \pm \text { SD (min) })^{11} \\
239.9 \pm 102.7 \text { vs. } 274.1 \pm \\
105.3, p<0.01\end{array}$ & & & & Unpaired $t$-test & 4 \\
\hline Rudzik et al., 2018 [43] & $\begin{array}{c}4,6,8,10,12,14,16,18 \\
\text { weeks }\end{array}$ & $\begin{array}{c}\text { EBF vs. FF } \\
\text { Actigraph report } \\
\text { EBF has } 55 \text { min-longer LSP } \\
\text { at } 18 \text { weeks, } p=0.04 \\
\text { No difference between } \\
\text { grps for LSP at } 4,6,8,10, \\
12,14,16 \text { weeks }\end{array}$ & & & & $t$-test & 3 \\
\hline Wailoo et al., 1990 [36] & 13-17 weeks & & $\begin{array}{c}\text { BF vs. FF } \\
\text { BF infants more likely to } \\
\text { disturb parents within } 4 \mathrm{~h}\left(\chi^{2}=\right. \\
5.9, \mathrm{DF} 3, p<0.01)\end{array}$ & & & $t$-test & 2 \\
\hline Huang et al., 2016 [39] & 0-34 weeks & & & & $\begin{array}{c}\mathrm{BF}+/- \text { food vs. FF +/- } \\
\text { food } \\
\text { No assoc. between LSP } \\
p>0.05\end{array}$ & Multilevel mixed models & 4 \\
\hline Mindell et al., 2012 [17] & $13-52$ weeks $^{4}$ & & & & $\begin{array}{c}\mathrm{BF}+/- \text { food vs. FF }+/- \\
\text { food } \\
\text { mean } \pm \mathrm{SD}(\mathrm{h}) \\
7.06 \pm 2.73 \text { vs. } 7.85 \pm 2.75 \\
p=0.249\end{array}$ & MANCOVA & 6 \\
\hline \multicolumn{8}{|c|}{ Sleep Onset Latency } \\
\hline \multirow{2}{*}{ Author, Year } & \multirow{2}{*}{$\begin{array}{c}\text { Infant Age at } \\
\text { Assessment/Assessment } \\
\text { Frequency }\end{array}$} & \multicolumn{2}{|c|}{$\leq 6$ Months } & $>6$ Months & 0-12 Months & \multirow{2}{*}{ Statistics ${ }^{1}$} & \multirow{2}{*}{ Quality Rating } \\
\hline & & EBF vs. FF $^{1}$ & BF vs. FF $^{1}$ & BF vs. FF $^{1}$ & BF vs. FF $^{1}$ & & \\
\hline Butte et al., 1992 [29] & 17 weeks & & $\begin{array}{c}\text { BF vs. FF } \\
\text { EEG: mean } \pm \text { SD (min) } \\
34.3 \pm 41.6 \text { vs. } 4.0 \pm 12.6 \\
p<0.05\end{array}$ & & & $\begin{array}{c}t \text {-test } \\
\text { Regression }\end{array}$ & 5 \\
\hline
\end{tabular}


Table 2. Cont.

\begin{tabular}{|c|c|c|c|c|c|c|}
\hline Figueiredo et al., 2017 [38] & 2, 13, 26 weeks & $\begin{array}{c}\text { EBF vs. FF } \\
\text { mean } \pm \text { SD }(h) \\
2 \text { weeks } \\
0.33 \pm 0.31 \text { vs. } 0.48 \pm 0.40 \\
p>0.05 \\
13 \text { weeks } \\
0.42 \pm 0.45 \text { vs. } 0.42 \pm 0.52 \\
p>0.05 \\
26 \text { weeks } \\
0.39 \pm 0.35 \text { vs. } 0.57 \pm 0.72 \\
p>0.05\end{array}$ & $\begin{array}{c}\text { BF vs. FF } \\
\text { mean } \pm \text { SD }(h) \\
2 \text { weeks } \\
0.56 \pm 0.75 \text { vs. } 0.48 \pm 0.40 \\
p>0.05 \\
13 \text { weeks } \\
0.44 \pm 0.41 \text { vs. } 0.42 \pm 0.52 \\
p>0.05 \\
26 \text { weeks } \\
0.51 \pm 0.31 \text { vs. } 0.57 \pm 0.72 \\
p>0.05\end{array}$ & & $\begin{array}{l}\text { Multivariate analyses of } \\
\text { Chi MANCOVA }^{3}\end{array}$ & 5 \\
\hline Tikotzky et al., 2011 [44] & 26 weeks & $\begin{array}{c}\text { EBF vs. FF } \\
\text { EBF assoc. with later sleep } \\
\text { onset }(\mathrm{r}=0.32, p<0.05)\end{array}$ & & & Spearman rho correlations & 6 \\
\hline Mindell et al., 2012 [17] & $13-52$ weeks $^{4}$ & & & $\begin{array}{c}\mathrm{BF}+/- \text { food vs. FF }+/- \\
\text { food } \\
\text { mean } \pm \mathrm{SD}(\mathrm{h}) \\
0.23 \pm 0.15 \text { vs. } 0.30 \pm 0.53 \\
p=0.427\end{array}$ & MANCOVA & 6 \\
\hline
\end{tabular}

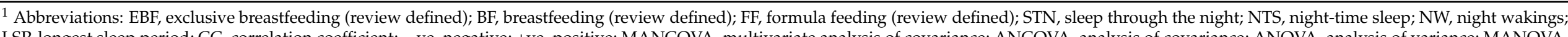

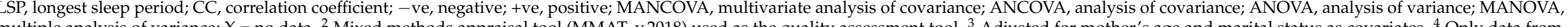

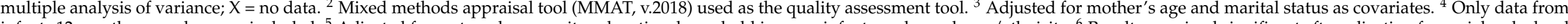

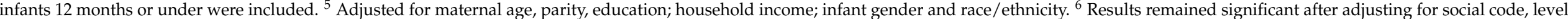

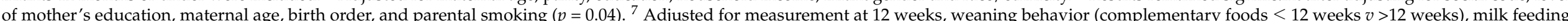

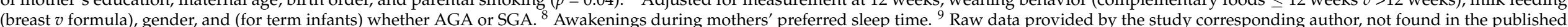
manuscript. ${ }^{10}$ Controlled for infant age and birth weight and maternal age and education. ${ }^{11}$ Longest sleep between 18:00 and 06:00. 
No association between night-waking frequency and breastfeeding compared to formula-fed infants older than six months was reported in one study [19]. Another study found breastfed infants to have a significantly greater frequency of night awakening compared to formula-fed infants [30].

In one study, no differences were found in night-waking frequency in infants aged 0-8 months who were breastfed or formula-fed [39]. However, breastfed infants had a significantly greater number of night-time wakings than formula-fed infants aged 3 to 12 months [17] and 2 to 12 months [35].

\subsubsection{Duration of Night Wakings}

Among infants aged 6 months and younger, no difference was reported in duration of night wakings between exclusively breastfed and formula-fed infants in one study [38]. However, another study reported a significantly longer duration of night wakings in exclusively breastfed infants compared to formula-fed infants [45].

Among infants older than 6 months, breastfeeding was reported to result in a significantly longer duration of night wakings compared to formula-fed infants in one study [30].

\subsubsection{Longest Sleep Period}

Two studies reported no difference in longest sleep period between exclusive breastfeeding and formula feeding among infants aged 6 months and younger [38,43]. In contrast, two studies $[32,38]$ found exclusively breastfed infants to have a significantly shorter longest sleep period compared to infants who were formula-fed. Of those studies, two studies measured the longest sleep period at multiple time points, one cohort [38] and another longitudinal design [43]. The longitudinal study [43] also found exclusively breastfed infants at 18 weeks-of-age to have a significantly longer longest sleep period as compared to formula-fed infants. Breastfed infants, when compared to formula-fed infants, reported no difference in longest sleep period in one study [38], while another study reported breastfed infants were more likely to wake parents in a four hour period than formula-fed infants [36].

No difference in longest sleep period duration was found in breastfed infants compared to formula-fed infants aged 3 to 12 months [17] and 0 to 8 months [39].

\subsubsection{Sleep Onset Latency}

Among infants aged 6 months and younger, one study reported no difference in sleep onset latency between exclusively breastfed and formula-fed infants [38], while another study reported exclusive breastfeeding was associated with later sleep onset time [44]. When breastfed infants were compared to formula-fed infants, no difference in sleep onset latency was reported in one study [38], while another study reported longer sleep onset duration associated with breastfed infants [29].

No difference in sleep onset latency was found in breastfed infants when compared to formula-fed infants aged 3 to 12 months [17].

\subsection{Introduction to Complementary Foods and Infant Sleep}

Four studies (two cohort, one combined RCT, and one cross-sectional) examined the association between the timing of introduction to complementary foods and infant sleep (Table 3). All sleep measurements were subjective, and information on timing of introduction to complementary foods was retrospectively collected from parents or caregivers. 
Table 3. Timing of introduction to complementary foods in relation to sleep variables including $24 \mathrm{~h}$ sleep duration and night-wakings frequency among infants 12 months-of-age and under.

\begin{tabular}{|c|c|c|c|c|}
\hline \multicolumn{5}{|c|}{24 h Sleep Duration } \\
\hline Author, Year & $\begin{array}{c}\text { Infant Age at } \\
\text { Assessment/Assessment } \\
\text { Frequency }\end{array}$ & $\begin{array}{l}\text { Sleep Outcomes on Introduction to } \\
\text { Complementary Foods }\end{array}$ & Statistics ${ }^{1}$ & Quality Rating ${ }^{2}$ \\
\hline Morgan et al., 2004 [46] & 9 months $^{3}$ & $\begin{array}{c}\leq 12 \text { weeks vs. }>12 \text { weeks } \\
\text { mean } \pm \text { SE }(\mathrm{h}) \\
11.4 \pm 0.1 \text { vs. } 11.2 \pm 0.1, p=0.07^{4}\end{array}$ & ANCOVA $^{5}$ & 5 \\
\hline Nevarez et al., 2010 [23] & 6,12 months $^{3}$ & $\begin{array}{c}<4 \text { months vs. } \geq 4 \text { months } \\
\text { Bivariate } \\
6 \text { months B }=-0.20 \text { ( } 95 \% \mathrm{CI}:-0.47 \text { to } \\
0.07), p>0.05 \\
12 \text { months } \mathrm{B}=-0.38(95 \% \mathrm{CI}:-0.64 \\
\text { to }-0.12), p<0.05 \\
\text { Multivariate } \\
6 \text { months B }=-0.05 \text { (95\%CI: }-0.35 \text { to } \\
0.24), p>0.05 \\
12 \text { months Is } \beta=-0.039(95 \% \mathrm{CI}: \\
-0.67 \text { to }-0.11), p<0.05\end{array}$ & $\begin{array}{l}\text { Bivariate } \\
\text { Multivariate linear } \\
\text { regression } 6\end{array}$ & 5 \\
\hline Heinig et al., 1993 [42] & 9 months & $\begin{array}{l}\quad<26 \text { weeks vs. } \geq 26 \text { weeks } \\
\text { mean } \pm \text { SE }(\mathrm{h}) \\
\text { BF group: } 12.2 \pm 1.1 \text { vs. } 12.1 \pm 1.0 \\
\qquad p>0.05 \\
\text { FF group: No associations between } \\
\text { age of solid food introduction and } \\
24 \text { h sleep duration }\end{array}$ & Regression & 4 \\
\hline \multicolumn{5}{|c|}{ Night-Waking Frequency } \\
\hline Author, Year & $\begin{array}{c}\text { Infant Age at } \\
\text { Assessment/Assessment } \\
\text { Frequency }\end{array}$ & $\begin{array}{l}\text { Sleep Outcomes on Introduction to } \\
\text { Complementary Foods }\end{array}$ & Statistics ${ }^{1}$ & Quality Rating $^{2}$ \\
\hline Brown et al., (2015) [19] & $6-12$ months & $\begin{array}{l}\text { Outcomes: } 8 \text { to } 32 \text { weeks (mean } \\
21.2 \text { weeks) } \\
\text { No associations between solid food } \\
\text { introduction and NW } \\
(\mathrm{r}=0.06, p=0.141)\end{array}$ & Pearson's CC & 5 \\
\hline
\end{tabular}

\footnotetext{
${ }^{1}$ Abbreviations: BF, breastfeeding; FF, formula feeding; NW, night wakings; CC, correlation coefficient; ANCOVA, analysis of covariance. ${ }^{2}$ mixed methods appraisal tool (MMAT, v.2018) used as the quality assessment tool. ${ }^{3}$ Only data from infants 12 months or under were included. ${ }^{4}$ Adjusted again for social code, level of mother's education, maternal age, birth order, and parental smoking. ${ }^{5}$ Adjusted for measurement at 12 weeks, weaning behavior (complementary foods $\leq 12$ weeks $v>12$ weeks), milk feeding (breast $v$ formula), gender, and (for term infants) whether AGA or SGA. ${ }^{6}$ Adjusted for maternal age, parity, education; household income; infant gender and race/ethnicity.
}

\subsection{1. $24 \mathrm{~h}$ Sleep Duration}

Three studies reported the relationship between the timing of introduction to complementary foods and $24 \mathrm{~h}$ sleep duration (Table 3). No difference was reported in $24 \mathrm{~h}$ sleep duration assessed at six [23] and nine [42,46] months-of-age among infants introduced to complementary foods at $\leq 12$ weeks (around 3 months) compared to at $>12$ weeks-of-age, at $<4$ months compared to at $\geq 4$ months-of-age, and at $<26$ weeks (at 6 months) compared to at $\geq 26$ weeks-of-age. However, infants slept $24 \mathrm{~min}$ less $(-0.39,95 \% \mathrm{CI}:-0.67--0.11)$ at 12 months-of-age if they were introduced to complementary foods at $<4$ months compared to at $\geq 4$ months-of-age [23].

Only two out of the four studies [42,46], examined the relationship between the timing of introduction to complementary foods and sleep among breastfed infants separately from formula-fed infants. Heinig et al. [42] reported similar results in both breastfed and formula-fed infants, where no difference in $24 \mathrm{~h}$ sleep duration was reported in relation to the timing of introduction to complementary foods. However, Morgan et al. [46] found that breastfed infants were more likely to sleep through the night at 9 months with early introduction of complementary foods at $\leq 12$ weeks as compared to at $>12$ weeks. This was not observed in formula-fed infants. However, the authors were unable to further 
their investigation due to the lack of data collected on the reasons for infants waking (i.e., waking for feeding or waking and self-soothing to sleep).

\subsubsection{Night-Waking Frequency}

One study of infants aged 6-12 months reported no significant association with night-waking frequency and timing of introduction to complementary foods (mean age of introduction 21 weeks); however, an association between later introduction to complementary foods and number of feeds during the night was reported (independent of infant age) [19].

\subsection{Quality Assessment}

All 21 studies were included in the quality assessment (Table 2). Quality scores ranged from 2 to 6 points (out of a total of 6 points). The mean quality score was 4 . The quality assessment variable with the lowest score was the additional question on the sleep assessment tool validation, where only 10 out of 21 studies reported using validated sleep assessment methods $[17,29,33-35,37,40,43-45]$. Four $(19 \%)$ studies $[17,35,37,44]$ had a quality score of 6 , whilst $14(66 \%)[19,23,29-32,34,38-42,45,46]$ scored $>4$ points, and 3 studies $(14 \%)[33,36,43]$ scored $\leq 3$ points. The three studies had low rating ( $\leq 3$ points) as their participants were not representative of the target population [33,36,43], had inappropriate outcome measurements [36], had less than 90\% completed data collection [33,43], did not account for confounders [33], the feeding group was not administered as intended [43], and did not use a validated sleep assessment tool [36].

\section{Discussion}

The main purpose of this systematic review was to examine the relationship between type of milk feeding, timing of introduction to complementary foods, and sleep in infants aged 12 months and younger. Among infants aged 6 months and younger, a majority of the studies (six out of nine studies, $67 \%$ ) reported no association between type of milk feeding and $24 \mathrm{~h}$ sleep duration. However, studies that examined infant sleep after 6 months-of-age (two out of three studies, 67\%) had a greater tendency to report less $24 \mathrm{~h}$ sleep duration among breastfed infants compared to formula-fed infants.

Among infants aged 6 months and younger, no significant difference in night-time sleep duration between different feeding types was reported by half of the studies included in the qualitative synthesis (five out of ten studies, 50\%), with two out of three (67\%) comparing exclusive breastfeeding to formula-feeding. In contrast, all studies that examined infant sleep after 6 months-of-age reported that breastfeeding was associated with less night-time sleep duration compared to formula-fed infants (two out of two studies), though the total number of studies was limited. Two studies $[33,43]$ classified as low-quality studies had quality ratings of 3 points and were excluded for sensitivity analysis. Removing these two studies from the qualitative synthesis did not change the overall conclusions on the relationship between type of milk feeding and night-time sleep.

Half (three out of six studies, $50 \%$ ) of the included studies reported formula-fed infants woke less often than exclusively breastfed infants aged 6 months and younger. This association could be explained by differences in rates of digestion of milk types (i.e., breast milk versus formula), contributing to shorter periods of satiety and a greater number of wakings $[48,49]$. Another mechanism proposed by Wolke et al., suggests differences in the number of sound signals created by breastfed infants versus formula-fed infants when awake may result in a greater number of identified and reported night wakings in breastfed infants [50]. In addition, breastfeeding mothers have been reported to have an increased sensitivity towards their infant's cry compared to formula-feeding mothers [51], potentially impacting the number of reported night wakings. In comparison, in five out of seven studies $(70 \%)$ reporting on breastfed versus formula-fed infants, no differences in the number of night wakings in breastfed infants 6 months-of-age and younger were found compared to formula-fed infants. The similarities in the number of night wakings could be 
attributed to the mixed consumption of formula and breast milk for both the breastfed and formula-fed infants. Three studies $[33,36,43]$ that reported on night-waking frequency had a low-quality rating ( $<3$ points), and removal of these studies from the qualitative synthesis strengthened the trend of more night wakings observed in exclusively breastfed infants, and no difference in night wakings was observed in breastfed infants in relation to formulafed infants. These results should be interpreted with caution and should not be used as a basis to change feeding practices, as the WHO recommends exclusive breastfeeding for the first six months of life as best practice for infants [2]. No clear conclusion could be made for type of milk feeding and frequency of night wakings after six months-of-age.

According to the qualitative synthesis, no associations were reported with the timing of introduction to complementary foods and sleep of infants younger than 12 months. Only one study [42] compared the introduction to complementary foods before and after 26 weeks (i.e., at 6 months-of-age), in accordance with WHO recommendations [2]. No longitudinal study has been carried out to assess infants' sleep before the introduction of complementary foods through to after the complementary feeding period in their first year of life. Conducting a longitudinal study is the most robust way of addressing the effects of timing of introduction to complementary foods defined by the WHO $(<6$ months vs. 6 months) [2] on sleep outcomes in later infancy and would be an important consideration for future research. The longitudinal design would also allow continuous monitoring of other important aspects of complementary feeding such as the volume and frequency of foods consumed in relation to infant sleep patterns.

\section{Strength and Limitations}

Findings from this systematic review are strengthened by the use of a comprehensive search strategy to capture all relevant studies in an extended database. Inclusion and exclusion criteria were well-defined in an effort to remove all studies that were not suited for this review. The use of a comprehensive search strategy, robust inclusion and exclusion criteria, assessment of study quality, and the absence of duplicate trial publications reduced the likelihood of publication bias. Half (6 out of 12) of the included studies that examined the association between feeding type and $24 \mathrm{~h}$ sleep duration and total night-time sleep, and more than half ( 8 out of 15 ) of the studies that examined the association between night-waking frequency and feeding type, reported no significant differences between milk feeding types, minimizing citation bias in this review.

However, there are several limitations. Firstly, there was significant heterogeneity in the type of assessment tools used for measuring infant sleep. Some studies only used subjective methods such as retrospective sleep questionnaires or sleep diaries [17,19,30-39,41,42,46], whilst other studies used objective measures such as an actigraph to capture real-time sleep data $[23,29,40,43-45]$. In addition, the quality of the assessment tool varied, as not all assessment tools were validated, with half $(50 \%)$ of the studies assessing sleep using non-validated, subjective assessment methods [19,23,30-32,36,38,39,41,42,46]. Furthermore, $<30 \%$ of the included studies used both objective and subjective sleep assessment tools [23,29,40,43-45]. Subjective sleep assessment tools are based on parental report, which increases reporting bias [52-54] through overestimating total sleep duration (i.e., placement in bed and time of rising) rather than true sleep and wake times determined by real-time measurements such as actigraphs. In addition, parental report may underestimate the number of night wakings [7] compared to actigraph-detected night wakings [54-56], as reported wakings are often associated with infant signals such as crying or calling for attention, rather than infant waking that is not associated with any sound $[53,54]$. Therefore, objective assessments such as actigraph measurements provide a more accurate estimate of sleep variables such as duration, sleep/wake time, and frequency of waking, especially when used in conjunction with a subjective method such as a sleep diary $[52,57]$.

Secondly, due to a limited number of included studies and inconsistent data reporting, we were unable to determine the effects of timing of introduction to complementary foods on infant sleep and the effects of type of milk feeding on sleep outcomes such as longest 
sleep period, sleep onset latency, and duration of night wakings. The lack of attention received for these sleep measures has also been reported by Dias et al. [7]. More studies are required to examine these sleep variables, especially sleep onset latency, a possible indicator of sleep quality [58].

Reported age of introduction to complementary foods varied widely across studies (mean age 21 weeks; $\leq 12$ weeks/ $>12$ weeks; $<26$ weeks $/ \geq 26$ weeks; $<4$ months $/ \geq 4$ months), with no standardized definitions for the collection of these data $[19,23,42,46]$. This heterogeneity made comparisons between studies challenging. Furthermore, conclusions from studies that examined the effects of early introduction of complementary foods (i.e., $<4$ months-ofage) $[23,46]$ or studies that examined the association between late introduction of solids (i.e., $\geq 7$ months) [19] must be interpreted with caution, as this does not align with current recommendations by the $\mathrm{WHO}[2]$.

Additionally, of the studies that examined infant milk feeding with and without the addition of complementary foods, none conducted separate analyses according to milk-feeding type with and without complementary food. These studies include infants 6 months and older, with no study examining the association between the volume and frequency of complementary foods consumed in relation to sleep outcomes. This could be a potential confounder since the addition of complementary foods and increase in the volume of food consumed between 6 to 12 months-of-age may influence infant sleep patterns.

Definitions of night-time and type of milk feeding were not well defined. The window of time considered 'night-time' varied, making the comparison of night-time sleep variables challenging. This lack of standardization of variables could account for the diverse range of sleep outcomes. Furthermore, some included studies did not clearly state whether the definition of breastfeeding included exclusive breastfeeding only, or a mixture of exclusively and partially breastfed infants, or infants that were predominantly breastfed. Though some studies have stated breastfeeding as 'exclusive" or "predominant', not all defined the terms are in accordance with the WHO definition, in which exclusive breastfeeding for the first 6 months refers to breast milk only with the exception of oral rehydration salts (ORS), drops, and syrups (vitamins, minerals, medicines) [27], while predominant breastfeeding includes the addition of liquids such as water and water-based drinks and fruit juice [27]. For example, Mindell et al. [17] and Huang et al. [39] have defined exclusive breastfeeding as breastmilk only with or without the inclusion of complementary foods among infants aged 3-12 months and 0-8 months, respectively. Therefore, sleep data reported after 6 months-of-age should be interpreted with caution, as complementary foods could impact sleep patterns.

Furthermore, in the qualitative synthesis for type of milk feeding and sleep, one longitudinal [43] and two cohort studies [38,39] assessed sleep at multiple time points, and had differing night-time sleep ranges, night-waking frequency, and longest sleep period outcomes at the different time points. An example of this is reported in the longitudinal study by Rudzik et al., where no association between type of milk feeding and night-time sleep was reported at most time points $(4,6,8,10,12,16$, and 18 weeks-of-age), with an association between breastfeeding and shorter sleep duration at 14 weeks-of-age [43].

Finally, most of the included studies were from western countries (North America and Europe), where there are variations in cultural practices associated with infant feeding compared to non-western countries. The inclusion of only English papers is a selection bias that could have contributed to the lack of cultural diversity in the studies included. Therefore, the data should be interpreted with caution and not applied to non-western populations. In addition, not all published studies were included in this review due to our strict inclusion and exclusion criteria. Criteria were strict in an effort to control for heterogeneity in the data. Therefore, the quality of the sleep data are limited to the quality of the individual studies included [59]. 


\section{Conclusions}

This is the first systematic literature review to compare the effects of type of milk feeding on selected sleep variables in infants under 12 months-of-age. Exclusively breastfed infants ( $\leq 6$ months-of-age) were reported more likely to wake at night compared to formula-fed infants, though this association was not found in breastfed infants (partial or predominantly breastfed). The majority of the studies reported no difference in night-time sleep duration and total $24 \mathrm{~h}$ sleep duration in both exclusively breastfed and breastfed infants ( $\leq 6$ months-of-age) compared to formula-fed infants. However, after 6 months-ofage, most studies reported breastfed infants to sleep less than formula-fed infants. Though studies were limited, the majority observed no association on the timing of introduction to complementary foods and total $24 \mathrm{~h}$ sleep duration, including one study that compared infants who were introduced complementary foods before and after 6 months-of-age in accordance with the WHO recommendations.

Further research should evaluate sleep variables such as longest sleep period and sleep onset latency, and most importantly, the effects of the timing of introduction to complementary food on sleep. There is a need for standardized, higher-quality sleep studies in this age group to address the heterogeneity in the type of assessment tool used, the definitions for type of milk feeding and night-time, and limited use of validated and objective tools for sleep assessment. This would provide a better understanding of the relationship between infant feeding and sleep. In addition, to truly understand the complexity between infant feeding (type of milk feeding and timing of introduction to complementary foods) and infant sleep, future research should adopt a longitudinal design, capturing sleep at transition time-points before and after changing feeding methods.

Supplementary Materials: The following are available online at https:/ /www.mdpi.com/article/10 $.3390 /$ nu13114105/s1, Table S1: Electronic database search strategy for systematic review on infant feeding mode and infant sleep. Table S2: Mixed Methods Appraisal Tool.

Author Contributions: Conceptualization, X.F., C.R.W., A.L.L., A.J.B. and R.F.M.; methodology, X.F. and A.L.L.; validation, C.R.W. and A.J.B.; writing-original draft, X.F.; writing-review and editing, X.F., C.R.W., A.L.L., A.J.B. and R.F.M. All authors have read and agreed to the published version of the manuscript.

Funding: This research received no external funding.

Institutional Review Board Statement: Not applicable.

Informed Consent Statement: Not applicable.

Data Availability Statement: Data sharing not applicable.

Conflicts of Interest: The authors declare no conflict of interest.

\section{References}

1. Hirshkowitz, M.; Whiton, K.; Albert, S.M.; Alessi, C.; Bruni, O.; DonCarlos, L.; Hazen, N.; Herman, J.; Hillard, P.J.A.; Katz, E.S.; et al National Sleep Foundation's updated sleep duration recommendations: Final report. Sleep Health 2015, 1, 233-243. [CrossRef] [PubMed]

2. World Health Organization. Infant and Young Child Feeding: Model Chapter for Textbooks for Medical Students and Allied Health Professionals; World Health Organization: Geneva, Switzerland, 2009.

3. León-Cava, N.; Lutter, C.; Ross, J.; Martin, L. Quantifying the Benefits of Breastfeeding: A Summary of the Evidence; Pan American Health Organization: Washington, DC, USA, 2002.

4. Horta, B.L.; Bahl, R.; Martinés, J.C.; Victora, C.G.; World Health Organization. Evidence on the Long-Term Effects of Breastfeeding: Systematic Review and Meta-Analyses; World Health Organization: Geneva, Switzerland, 2007.

5. Henderson, J.M.T.; France, K.G.; Owens, J.L.; Blampied, N.M. Sleeping through the Night: The Consolidation of Self-regulated Sleep across the First Year of Life. Pediatrics 2010, 126, e1081-e1087. [CrossRef] [PubMed]

6. Davis, K.F.; Parker, K.P.; Montgomery, G.L. Sleep in infants and young children: Part one: Normal sleep. J. Pediatr. Health Care 2004, 18, 65-71. [CrossRef]

7. Dias, C.C.; Figueiredo, B.; Rocha, M.; Field, T. Reference values and changes in infant sleep-wake behaviour during the first 12 months of life: A systematic review. J. Sleep Res. 2018, 27, e12654. [CrossRef] [PubMed] 
8. Tham, E.K.; Schneider, N.; Broekman, B.F. Infant sleep and its relation with cognition and growth: A narrative review. Nat. Sci. Sleep 2017, 9, 135-149. [CrossRef]

9. Seehagen, S.; Konrad, C.; Herbert, J.; Schneider, S. Timely sleep facilitates declarative memory consolidation in infants. Proc. Natl. Acad. Sci. USA 2015, 112, 1625-1629. [CrossRef]

10. Friedrich, M.; Wilhelm, I.; Born, J.; Friederici, A.D. Generalization of word meanings during infant sleep. Nat. Commun. 2015, 6, 6004. [CrossRef] [PubMed]

11. Taveras, E.M.; Rifas-Shiman, S.L.; Oken, E.; Gunderson, E.P.; Gillman, M.W. Short Sleep Duration in Infancy and Risk of Childhood Overweight. Arch. Pediatr. Adolesc. Med. 2008, 162, 305-311. [CrossRef] [PubMed]

12. Zuckerman, B.; Stevenson, J.; Bailey, V. Sleep Problems in Early-Childhood-Continuities, Predictive Factors, and Behavioral Correlates. Pediatrics 1987, 80, 664-671.

13. Martin, J.; Hiscock, H.; Hardy, P.; Davey, B.; Wake, M. Adverse Associations of Infant and Child Sleep Problems and Parent Health: An Australian Population Study. Pediatrics 2007, 119, 947-955. [CrossRef]

14. Bernier, A.; Carlson, S.M.; Bordeleau, S.; Carrier, J. Relations Between Physiological and Cognitive Regulatory Systems: Infant Sleep Regulation and Subsequent Executive Functioning. Child Dev. 2010, 81, 1739-1752. [CrossRef] [PubMed]

15. Karraker, K.H.; Young, M. Night waking in 6-month-old infants and maternal depressive symptoms. J. Appl. Dev. Psychol. 2007, 28, 493-498. [CrossRef] [PubMed]

16. Hiscock, H.; Wake, M. Infant sleep problems and postnatal depression: A community-based study. Pediatrics 2001, 107, 1317-1322. [CrossRef] [PubMed]

17. Teng, A.; Bartle, A.; Sadeh, A.; Mindell, J. Infant and toddler sleep in Australia and New Zealand. J. Paediatr. Child Health 2011, 48, 268-273. [CrossRef]

18. Galbally, M.; Lewis, A.J.; McEgan, K.; Scalzo, K.; Islam, F.A. Breastfeeding and infant sleep patterns: An Australian population study. J. Paediatr. Child Health 2013, 49, E147-E152. [CrossRef] [PubMed]

19. Brown, A.; Harries, V. Infant Sleep and Night Feeding Patterns During Later Infancy: Association with Breastfeeding Frequency, Daytime Complementary Food Intake, and Infant Weight. Breastfeed. Med. 2015, 10, 246-252. [CrossRef] [PubMed]

20. Perkin, M.R.; Bahnson, H.T.; Logan, K.; Marrs, T.; Radulovic, S.; Craven, J.; Flohr, C.; Lack, G. Association of Early Introduction of Solids with Infant Sleep. JAMA Pediatr. 2018, 172, e180739. [CrossRef] [PubMed]

21. Dias, C.C.; Figueiredo, B. Sleep-wake behaviour during the first 12 months of life and associated factors: A systematic review. Early Child Dev. Care 2020, 190, 2333-2365. [CrossRef]

22. Demirci, J.R.; Braxter, B.J.; Chasens, E.R. Breastfeeding and short sleep duration in mothers and 6-11-month-old infants. Infant Behav. Dev. 2012, 35, 884-886. [CrossRef] [PubMed]

23. Nevarez, M.D.; Rifas-Shiman, S.L.; Kleinman, K.; Gillman, M.W.; Taveras, E.M. Associations of Early Life Risk Factors with Infant Sleep Duration. Acad. Pediatr. 2010, 10, 187-193. [CrossRef]

24. Alder, E.M.; Williams, F.L.R.; Anderson, A.S.; Forsyth, S.; Florey, C.D.V.; Van Der Velde, P. What influences the timing of the introduction of solid food to infants? Br. J. Nutr. 2004, 92, 527-531. [CrossRef]

25. Moher, D.; Liberati, A.; Tetzlaff, J.; Altman, D.G. Preferred Reporting Items for Systematic Reviews and Meta-Analyses: The PRISMA Statement. J. Clin. Epidemiol. 2009, 62, 1006-1012. [CrossRef]

26. Clarivate. The EndNote Team EndNote, EndNote X9; Clarivate: Philadelphia, PA, USA, 2013.

27. World Health Organization. Indicators for Assessing Infant and Young Child Feeding Practices Part 3: Country Profiles; World Health Organization: Geneva, Switzerland, 2010.

28. Hong, Q.N.; Fàbregues, S.; Bartlett, G.; Boardman, F.; Cargo, M.; Dagenais, P.; Gagnon, M.-P.; Griffiths, F.; Nicolau, B.; O'Cathain, A.; et al. The Mixed Methods Appraisal Tool (MMAT) version 2018 for information professionals and researchers. Educ. Inf. 2018, 34, 285-291. [CrossRef]

29. Butte, N.F.; Jensen, C.L.; Moon, J.K.; Glaze, D.G.; Frost, J.D. Sleep Organization and Energy Expenditure of Breast-Fed and Formula-Fed Infants. Pediatr. Res. 1992, 32, 514-519. [CrossRef] [PubMed]

30. DeLeon, C.W.; Karraker, K.H. Intrinsic and extrinsic factors associated with night waking in 9-month-old infants. Infant Behav. Dev. 2007, 30, 596-605. [CrossRef]

31. Kaley, F.; Reid, V.; Flynn, E. Investigating the biographic, social and temperamental correlates of young infants' sleeping, crying and feeding routines. Infant Behav. Dev. 2012, 35, 596-605. [CrossRef] [PubMed]

32. Lee, K. Crying and behavior pattern in breast- and formula-fed infants. Early Hum. Dev. 2000, 58, 133-140. [CrossRef]

33. Quillin, S.I.M. Infant and mother sleep patterns during 4th postpartum week. Issues Compr. Pediatr. Nurs. 1997, 20, 115-123. [CrossRef] [PubMed]

34. Quillin, I.; Glenn, L.L. Interaction Between Feeding Method and Co-Sleeping on Maternal-Newborn Sleep. J. Obstet. Gynecol. Neonatal Nurs. 2004, 33, 580-588. [CrossRef] [PubMed]

35. Sun, W.; Li, S.X.; Jiang, Y.; Xu, X.; Spruyt, K.; Zhu, Q.; Tseng, C.-H.; Jiang, F. A Community-Based Study of Sleep and Cognitive Development in Infants and Toddlers. J. Clin. Sleep Med. 2018, 14, 977-984. [CrossRef] [PubMed]

36. Wailoo, M.P.; Petersen, S.A.; Whitaker, H. Disturbed nights and 3-4 month old infants: The effects of feeding and thermal environment. Arch. Dis. Child. 1990, 65, 499-501. [CrossRef]

37. Berger, P.K.; Lavner, J.A.; Smith, J.J.; Birch, L.L. Differences in early risk factors for obesity between African American formula-fed infants and White breastfed controls. Pilot Feasibility Stud. 2017, 3, 1-8. [CrossRef] [PubMed] 
38. Figueiredo, B.; Dias, C.C.; Pinto, T.M.; Field, T. Exclusive breastfeeding at three months and infant sleep-wake behaviors at two weeks, three and six months. Infant Behav. Dev. 2017, 49, 62-69. [CrossRef] [PubMed]

39. Huang, X.-N.; Wang, H.-S.; Chang, J.-J.; Wang, L.-H.; Liu, X.-C.; Jiang, J.-X.; An, L. Feeding methods, sleep arrangement, and infant sleep patterns: A Chinese population-based study. World J. Pediatr. 2016, 12, 66-75. [CrossRef]

40. Tikotzky, L.; Sadeh, A.; Volkovich, E.; Manber, R.; Meiri, G.; Shahar, G. VII. infant sleep development from 3 to 6 months postpartum: Links with maternal sleep and paternal involvement. Monogr. Soc. Res. Child Dev. 2015, 80, 107-124. [CrossRef] [PubMed]

41. Pennestri, M.-H.; Laganière, C.; Bouvette-Turcot, A.-A.; Pokhvisneva, I.; Steiner, M.; Meaney, M.J.; Gaudreau, H.; on behalf of the Mavan Research Team. Uninterrupted Infant Sleep, Development, and Maternal Mood. Pediatrics 2018, 142, e20174330. [CrossRef] [PubMed]

42. Heinig, M.J.; Nommsen, L.A.; Peerson, J.M.; Lonnerdal, B.; Dewey, K.G. Intake and growth of breast-fed and formula-fed infants in relation to the timing of introduction of complementary foods: The DARLING study. Acta Paediatr. 1993, 82, 999-1006. [CrossRef]

43. Rudzik, A.E.; Robinson-Smith, L.; Ball, H.L. Discrepancies in maternal reports of infant sleep vs. actigraphy by mode of feeding. Sleep Med. 2018, 49, 90-98. [CrossRef]

44. Tikotzky, L.; Sadeh, A.; Glickman-Gavrieli, T. Infant Sleep and Paternal Involvement in Infant Caregiving During the First 6 Months of Life. J. Pediatr. Psychol. 2010, 36, 36-46. [CrossRef] [PubMed]

45. Yoshida, M.; Shinohara, H.; Kodama, H. Assessment of nocturnal sleep architecture by actigraphy and one-channel electroencephalography in early infancy. Early Hum. Dev. 2015, 91, 519-526. [CrossRef]

46. Morgan, J.B.; Lucas, A.; Fewtrell, M.S. Does weaning influence growth and health up to 18 months? Arch. Dis. Child. 2004, 89, 728-733. [CrossRef]

47. Sadeh, A. A Brief Screening Questionnaire for Infant Sleep Problems: Validation and Findings for an Internet Sample. Pediatrics 2004, 113, e570-e577. [CrossRef] [PubMed]

48. Touchette, É.; Petit, D.; Paquet, J.; Boivin, M.; Japel, C.; Tremblay, R.E.; Montplaisir, J.Y. Factors Associated with Fragmented Sleep at Night Across Early Childhood. Arch. Pediatr. Adolesc. Med. 2005, 159, 242-249. [CrossRef] [PubMed]

49. Lanting, C.; Huisman, M.; Boersma, E.; Touwen, B.; Fidler, V. Neurological differences between 9-year-old children fed breast-milk or formula-milk as babies. Lancet 1994, 344, 1319-1322. [CrossRef]

50. Wolke, D.; Bilgin, A.; Samara, M. Systematic Review and Meta-Analysis: Fussing and Crying Durations and Prevalence of Colic in Infants. J. Pediatr. 2017, 185, 55-61.e4. [CrossRef] [PubMed]

51. Kim, P.; Feldman, R.; Mayes, L.C.; Eicher, V.; Thompson, N.; Leckman, J.F.; Swain, J.E. Breastfeeding, brain activation to own infant cry, and maternal sensitivity. J. Child Psychol. Psychiatry 2011, 52, 907-915. [CrossRef]

52. Gozal, D.; Dayyat, E.A.; Spruyt, K.; Molfese, D.L. Sleep estimates in children: Parental versus actigraphic assessments. Nat. Sci. Sleep 2011, 3, 115-123. [CrossRef]

53. Galland, B.C.; Taylor, B.; Elder, D.E.; Herbison, P. Normal sleep patterns in infants and children: A systematic review of observational studies. Sleep Med. Rev. 2012, 16, 213-222. [CrossRef]

54. Hall, W.A.; Eliva, S.; Emoynihan, M.; Esaunders, R. A Comparison of Actigraphy and Sleep Diaries for Infants' Sleep Behavior. Front. Psychiatry 2015, 6, 19. [CrossRef] [PubMed]

55. Iwasaki, M.; Iwata, S.; Iemura, A.; Yamashita, N.; Tomino, Y.; Anme, T.; Yamagata, Z.; Iwata, O.; Matsuishi, T. Utility of Subjective Sleep Assessment Tools for Healthy Preschool Children: A Comparative Study Between Sleep Logs, Questionnaires, and Actigraphy. J. Epidemiol. 2010, 20, 143-149. [CrossRef]

56. Bauer, K.; Blunden, S. How Accurate is Subjective Reporting of Childhood Sleep Patterns? A Review of the Literature and Implications for Practice. Curr. Pediatr. Rev. 2008, 4, 132-142. [CrossRef]

57. Sadeh, A. Evaluating Night Wakings in Sleep-Disturbed Infants: A Methodological Study of Parental Reports and Actigraphy. Sleep 1996, 19, 757-762. [CrossRef] [PubMed]

58. Ohayon, M.; Wickwire, E.M.; Hirshkowitz, M.; Albert, S.; Avidan, A.; Daly, F.J.; Dauvilliers, Y.; Ferri, R.; Fung, C.; Gozal, D.; et al. National Sleep Foundation's sleep quality recommendations: First report. Sleep Health 2017, 3, 6-19. [CrossRef]

59. Downs, S.H.; Black, N. The feasibility of creating a checklist for the assessment of the methodological quality both of randomised and non-randomised studies of health care interventions. J. Epidemiol. Community Health 1998, 52, 377-384. [CrossRef] 